$04,05,10$

\title{
Электронная структура, динамика решетки и магнитоэлектрические свойства двойного перовскита $\mathrm{La}_{2} \mathrm{CuTiO}_{6}$
}

\author{
() В.И. Зиненко, М.С. Павловский, А.С. Шинкоренко \\ Институт физики им. Л.В. Киренского СО РАН, \\ Красноярск, Россия \\ E-mail: zvi@iph.krasn.ru
}

(Поступила в Редакцию 17 марта 2016 г.)

Приводятся результаты ab initio расчетов электронной структуры, колебательных свойств и магнитоэлектрического эффекта в кристалле $\mathrm{La}_{2} \mathrm{CuTiO}_{6}$ со структурой двойного перовскита. Расчет динамики решетки показал наличие нестабильных мод в фононном спектре высокосимметричной кубической фазы с пространственной группой симметрии $F m \overline{3} m$. Конденсация двух наиболее нестабильных мод, принадлежащих центру и граничной точке $X$ зоны Бриллюэна, приводит к возникновению неполярной стабильной фазы с пространственной группой симметрии $P 2_{1} / n$. Расчет с учетом спиновой поляризации показал, что основное магнитное состояние является антиферромагнитным $E^{*}$-типа с удвоением магнитной ячейки и с конфигурацией магнитных моментов ионов меди типа два спина вверх, два спина вниз вдоль кристаллографического направления [010]. Такое упорядочение магнитных моментов приводит к полярной пространственной группе и появлению поляризации. Оценка величины поляризации дает значение $71 \mu \mathrm{C} / \mathrm{m}^{2}$.

Работа поддержана грантом РФФИ № 15-02-00340-а и грантом Президента РФ „Ведушие научные школы“.

Расчеты проводились с использованием компьютерных ресурсов НИЦ „Курчатовский институт“ (ui2.computing.kiae.ru).

\section{1. Введение}

Двойные перовскиты с общей формулой $A A^{\prime} B B^{\prime} \mathrm{O}_{6}$ исследуются в течение нескольких десятилетий из-за разнообразия их физических свойств, которые зависят как от химического состава катионов $A, A^{\prime}, B$ и $B^{\prime}$, так и от различных типов и степени упорядочения катионов в структуре. В настоящее время существует большое число экспериментальных и теоретических работ, посвященных исследованию соединений, принадлежащих к семейству двойных перовскитов [1-5]. Соединения, полностью разупорядоченные по катионам $A, A^{\prime}$ и $B$, $B^{\prime}$, обладают структурой перовскита и в неискаженной фазе имеют пространственную группу симметрии $P m \overline{3} m$. Соединения $A A^{\prime} B B^{\prime} \mathrm{O}_{6}$ и $A_{2} B B^{\prime} \mathrm{O}_{6}$, упорядоченные по катионам вдоль направления [111], также обладают кубической симметрией с пространственными группами $F \overline{4} 3 m$ и $F m \overline{3} m$ соответственно. В большинстве двойных перовскитов (за редким исключением) вне зависимости от степени упорядочения катионов в кубической фазе имеются неустойчивости, связанные с модами колебаний решетки, собственные векторы которых соответствуют „поворотам“ октаэдров $B \mathrm{O}_{6}$. В последнее время внимание исследователей привлекают окислы со структурой двойного перовскита, содержащие в своем составе элементы переходных или редкоземельных металлов. В некоторых из этих соединений наряду с искажениями кристаллической решетки, связанными с ,поворотом“ октаэдра $B \mathrm{O}_{6}$, существуют конкурирующие взаимодействия между магнитными ионами. Такие взаимодействия могут приводить к упорядоченным магнитным структу- рам, в которых теряется центр инверсии и соответственно в результате магнитного упорядочения возникает спонтанная поляризация.

Соединение $\mathrm{La}_{2} \mathrm{CuTiO}_{6}$ (LCTO) известно уже более двадцати лет $[6,7]$, однако сведения о его физических свойствах немногочисленны. Структурные исследования показывают, что структура кристалла представляет собой искаженную форму кубического двойного перовскита с пространственной группой симметрии Pbnm и с двумя молекулами в элементарной ячейке [6-8]. При этом позиции $B$ и $B^{\prime}$ заняты ионами $\mathrm{Cu}$ и $\mathrm{Ti}$ с равной вероятностью, т.е., согласно результатам [6-8], кристалл остается разупорядоченным по этим катионам вплоть до низких температур. В результате исследования температурной зависимости магнитной восприимчивости LCTO [8] показано, что соединение испытывает фазовый переход в антиферромагнитное состояние с парамагнитной температурой Кюри $-38 \mathrm{~K}$, однако тип антиферромагнитного состояния в этой работе не приводится. Сведений о свойствах LCTO, упорядоченного по катионам $\mathrm{Cu}$ и $\mathrm{Ti}$, нами в литературе не обнаружено, хотя изучение свойств упорядоченного кристалла, которые могут существенным образом отличаться от свойств разупорядоченного кристалла, представляет интерес.

В настоящей работе приводятся результаты $a b$ initio расчета электронной структуры, динамики кристаллической решетки, магнитных свойств и магнитоэлектрического эффекта кристалла LCTO, упорядоченного в направлении, соответствующем направлению [111] в структуре перовскита. 
Таблица 1. Параметры решетки и относительные координаты атомов LCTO в рассматриваемых фазах, полученные в результате расчета (в скобках указаны экспериментальные данные для разупорядоченного LCTO в фазе с пространственной группой симметрии Рbnm [6])

\begin{tabular}{|c|c|c|c|c|c|c|}
\hline $\begin{array}{c}\text { Группа } \\
\text { симметрии }\end{array}$ & Атом & $\begin{array}{l}\text { Позиция } \\
\text { Уайкова }\end{array}$ & $x / a$ & $y / b$ & $z / c$ & $\begin{array}{c}\text { Параметры } \\
\text { решетки }\end{array}$ \\
\hline$F m \overline{3} m$ & $\begin{array}{c}\mathrm{La} \\
\mathrm{Cu} \\
\mathrm{Ti} \\
\mathrm{O}\end{array}$ & $\begin{array}{c}8 c \\
4 a \\
4 b \\
24 e\end{array}$ & $\begin{array}{l}0.25000 \\
0.00000 \\
0.50000 \\
0.25481\end{array}$ & $\begin{array}{l}0.25000 \\
0.00000 \\
0.50000 \\
0.00000\end{array}$ & $\begin{array}{l}0.25000 \\
0.00000 \\
0.50000 \\
0.00000\end{array}$ & $a=7.86928 \AA$ \\
\hline$P 2_{1} / n$ & $\begin{array}{l}\mathrm{La} \\
\mathrm{Cu} \\
\mathrm{Ti} \\
\mathrm{O} 1 \\
\mathrm{O} 2 \\
\mathrm{O} 3\end{array}$ & $\begin{array}{l}4 e \\
2 c \\
2 d \\
4 e \\
4 e \\
4 e\end{array}$ & $\begin{array}{c}-0.00909 \\
(-0.00970) \\
0.50000 \\
0.50000 \\
0.08474 \\
(0.07593) \\
0.68813 \\
(0.71161) \\
0.21442\end{array}$ & $\begin{array}{c}-0.05429 \\
(-0.03870) \\
0.00000 \\
0.00000 \\
0.52654 \\
(0.51703) \\
0.71408 \\
(0.71088) \\
0.81541\end{array}$ & $\begin{array}{c}0.24966 \\
(0.25000) \\
0.50000 \\
0.00000 \\
0.24737 \\
(0.25000) \\
0.04539 \\
(0.04000) \\
-0.04414\end{array}$ & $\begin{array}{c}a=5.64082 \AA \\
(a=5.5702 \AA) \\
b=5.85075 \AA \\
(b=5.6262 \AA) \\
c=7.70505 \AA \\
(c=7.8396 \AA) \\
\alpha=90^{\circ} \\
\beta=90^{\circ} \\
\gamma=90^{\circ}\end{array}$ \\
\hline
\end{tabular}

\section{2. Метод расчета}

Расчеты проведены в рамках теории функционала плотности с использованием обменно-корреляционных функционалов Пердью-Бурка-Эрнцерхофа с обобщенной градиентной аппроксимацией (PBE-GGA), реализованных в пакете VASP („The Vienna Ab initio Simulation Package ${ }^{\text {“ }}$ [9,10]. В расчете был использован метод $\mathrm{GGA}+U$ в приближении Дударева [11], где значение $U$ для ионов меди и титана бралось равным $4 \mathrm{eV}$. Расчеты были проведены как без учета, так и с учетом спиновой поляризации. Для расчета магнитного основного состояния и обменных констант в низкосимметричной фазе использовались суперъячейки, содержащие 40 атомов. Сетка Монкхорста-Пака выбиралась равной $6 \times 6 \times 6$. Оптимизация параметров решетки и координат ионов проводилась до тех пор, пока остаточные силы, действующие на ионы, не становились меньше $0.02 \mathrm{eV} / \AA$. Обменные константы гамильтониана Гейзенберга были рассчитаны по разности энергий между упорядоченными магнитными конфигурациями.

\section{3. Результаты и обсуждение}

3.1. Структурные и колебательные свойства. Расчеты структурных и колебательных свойств проведены без учета спиновой поляризации. Высокосимметричная фаза LCTO c „шахматным“ упорядочением катионов $\mathrm{Cu}$ и Ті обладает кубической симметрией с пространственной группой $F m \overline{3} m$ и с одной молекулой в элементарной ячейке (рис. 1). Оптимизированные значения параметра решетки и координат атомов в кубической фазе представлены в табл. 1 .

Вычисленные значения частот колебаний кристаллической решетки в центре зоны Бриллюэна и их клас- сификация по неприводимым представлениям указаны в табл. 2. Как видно из этой таблицы, в центре зоны Бриллюэна исследуемого соединения присутствуют две нестабильные моды колебания $F_{1 u}$ и $F_{1 g}$. Наиболее неустойчивой является трехкратно вырожденная мода $F_{1 g}$, в собственных векторах которой смещаются только ионы кислорода. Эти смещения соответствуют „поворотам“ октаэдра $\mathrm{TiO}_{6}\left(a^{-}, b^{-}, c^{-}\right.$в обозначениях Глэзеpa [12]). Мода $F_{1 u}$ является полярной модой и связана c сегнетоэлектрической неустойчивостью кубической фазы. Следует подчеркнуть, что наряду с неустойчивостями в центре зоны Бриллюэна в кристалле имеются однократные мягкие моды колебаний в граничных точках $X, Y$ и $Z$ типа $X_{5}, Y_{5}$ и $Z_{5}$ соответственно. Частота

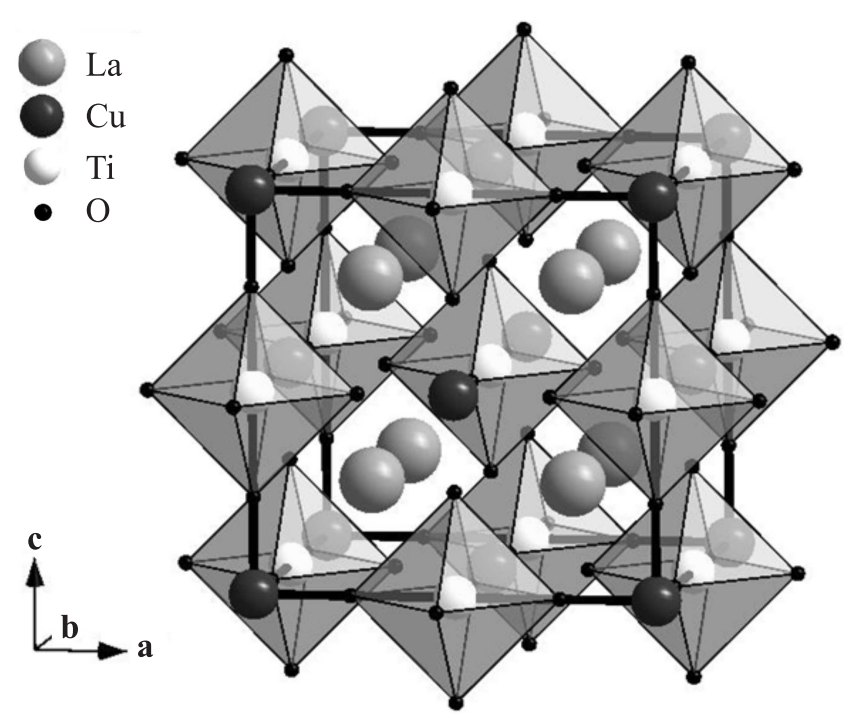

Рис. 1. Структура LCTO с „шахматным“ упорядочением катионов $\mathrm{Cu}$ и Ti, пространственная группа симметрии $F m \overline{3} m$. 
Таблица 2. Вычисленные частоты колебаний $\left(\mathrm{cm}^{-1}\right)$ кристаллической решетки LCTO в центре зоны Бриллюэна в исследуемых фазах (разложение полного колебательного представления имеет вид $\Gamma=A_{1 g}+E_{g}+F_{1 g}+2 F_{2 g}+5 F_{1 u}+F_{2 u}$, куда входят акустические колебания $F_{1 u}$, для кубической фазы; $\Gamma=12 A_{g}+12 B_{g}+18 A_{u}+18 B_{u}$, куда входят акустические колебания $A_{u}+2 B_{u}$, для моноклинной фазы)

\begin{tabular}{|c|c|c|c|c|c|c|c|c|c|}
\hline \multicolumn{6}{|c|}{ Кубическая фаза $(F m \overline{3} m)$} & \multicolumn{4}{|c|}{ Моноклинная фаза $\left(P 2_{1} / n\right)$} \\
\hline$A_{1 g}$ & $E_{g}$ & $F_{1 g}$ & $F_{2 g}$ & $F_{1 u}$ & $F_{2 u}$ & $A_{g}$ & $B_{g}$ & $A_{u}$ & $B_{u}$ \\
\hline 772 & 404 & $235 i$ & $\begin{array}{r}360 \\
57\end{array}$ & $\begin{array}{c}643 \\
363 \\
237 \\
70 i\end{array}$ & 131 & $\begin{array}{r}635 \\
458 \\
411 \\
401 \\
322 \\
320 \\
292 \\
244 \\
194 \\
140 \\
120 \\
98\end{array}$ & $\begin{array}{r}617 \\
479 \\
454 \\
406 \\
344 \\
331 \\
275 \\
202 \\
177 \\
167 \\
134 \\
99\end{array}$ & $\begin{array}{r}514 \\
495 \\
469 \\
456 \\
406 \\
356 \\
330 \\
309 \\
271 \\
249 \\
200 \\
189 \\
174 \\
153 \\
150 \\
91 \\
86\end{array}$ & $\begin{array}{r}518 \\
508 \\
460 \\
428 \\
384 \\
352 \\
349 \\
309 \\
283 \\
259 \\
211 \\
177 \\
159 \\
149 \\
132 \\
99\end{array}$ \\
\hline
\end{tabular}

каждой такой моды $\left(225 \mathrm{~cm}^{-1}\right)$ близка по величине к частоте моды $F_{1 g}$, и их собственные векторы также соответствуют ,повороту“ октаэдра $\mathrm{TiO}_{6}\left(a^{+}, b^{+}, c^{+}\right.$в обозначениях Глэзера [12]). В большинстве двойных перовскитов $A_{2} B B^{\prime} \mathrm{O}_{6}$ с трехвалентным ионом в позиции $A$ среди возможных фаз с искажениями, связанными с собственными векторами мод $F_{1 g}, X_{5}, Y_{5}, Z_{5}$, наиболее выгодной по энергии является низкосимметричная фаза с искажением $\left(a^{-}, a^{-}, c^{+}\right)$. Для соединений, неупорядоченных и упорядоченных по катионам $B B^{\prime}$, такое искажение приводит к пространственным группам Pbnm и $P 2_{1} / n$ соответственно. Для исследуемого в настоящей работе соединения задавались малые смещения ионов кислорода по собственным векторам мод $F_{1 g}$ и $X_{5}$ $(u=0.01)$ относительно позиций в кубической фазе с последующей релаксацией структуры. Полученные в результате оптимизированные параметры ячейки и координаты атомов для LCTO в фазе $P 2_{1} / n$ представлены в табл. 1. Искажения в структуре связаны в основном с „поворотами“ октаэдра $\mathrm{TiO}_{6}$ на приблизительно равные значения углов „поворота““ $\left(\sim 11^{\circ}\right)$ для $a^{-}$и $c^{+}$. Отметим, что это значение угла „поворота“ неплохо согласуется со значением угла „поворота“ $\left(\sim 9^{\circ}\right)$, вычисленным из экспериментально определенных координат атомов LCTO в фазе Pbnm с полным разупорядочением ионов меди и титана (табл. 1). Вычисленные частоты предельных колебаний решетки LCTO в фазе $P 21 / n$ представлены в табл. 2. Как видно из этой таблицы, в моноклинной фазе все предельные моды колебаний стабильны, т.е. сегнетоэлектрическая неустойчивость, присутствующая в кубической фазе, существенно подавляется антиферродисторсионными искажениями решетки (частота наиболее низкой по величине полярной моды в фазе $P 2_{1} / n$ равна $\left.86 \mathrm{~cm}^{-1}\right)$.

3.2. Электронная структура. Электронная структура $\mathrm{La}_{2} \mathrm{CuTiO}_{6}$ вычислена как с учетом, так и без учета спиновой поляризации. Результаты представлены на рис. 2-5. На рис. 2 показана вычисленная без учета спиновой поляризации зонная структура вдоль симмет-

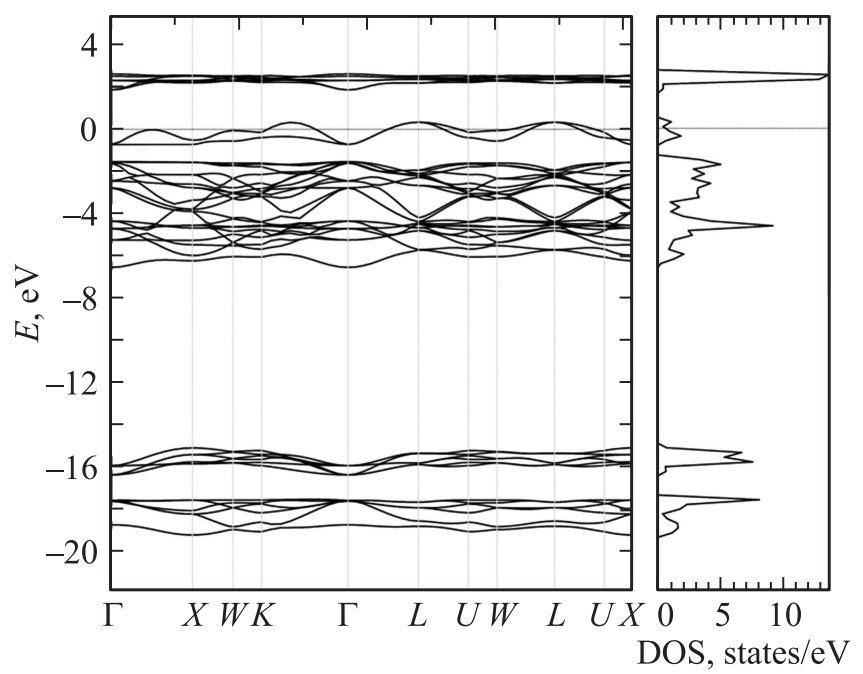

Pис. 2. Электронная зонная структура и плотность состояний (DOS) кристалла LCTO в кубической фазе с пространственной группой симметрии $F m \overline{3} m$. 
ричных направлений зоны Бриллюэна кубической фазы $F m \overline{3} m$. Там же показана полная плотность электронных состояний. На рис. 3 показано разложение парциальных плотностей состояний для $\mathrm{La}, \mathrm{Cu}, \mathrm{Ti}$ и О по орбитальным моментам. Как видно из этих рисунков, две зоны, образованные $p$-электронами кислорода и $d$-электронами титана и меди, в валентной зоне находятся вблизи и частично заполняют уровень Ферми. Результат расчета зонной структуры в моноклинной фазе с удвоенной элементарной ячейкой без учета спиновой поляризации качественно не отличается от приведенного на рис. 2 и здесь не приводится. На рис. 4 показан результат расчета зонной структуры LCTO с учетом спиновой поляризации в моноклинной фазе $P 2_{1} / n$ для ячейки, содержащей четыре молекулы LCTO (такая ячейка соответствует

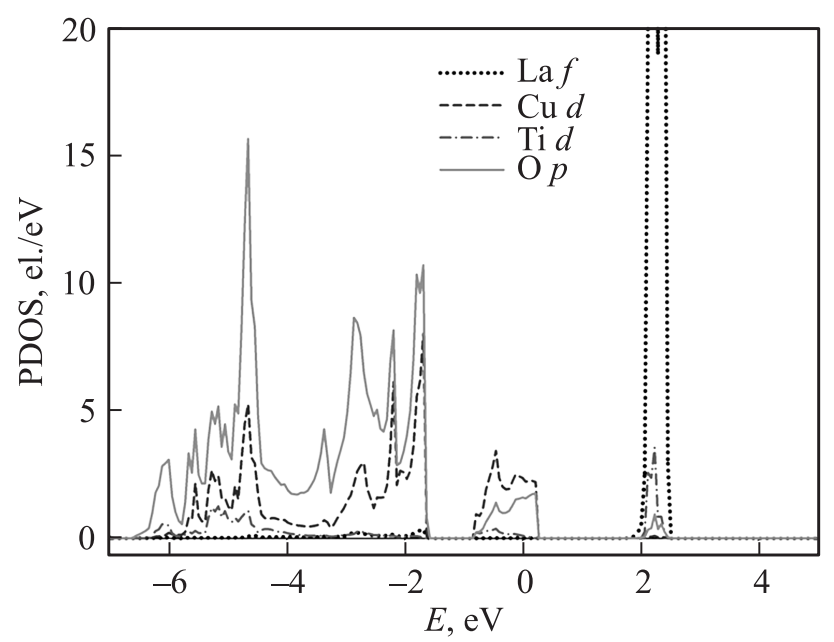

Pис. 3. Разложение парциальных плотностей состояний $(\mathrm{PDOS})$ для $\mathrm{La}, \mathrm{Cu}, \mathrm{Ti}$ и O по орбитальным моментам в фазе $F m \overline{3} m$ LCTO.

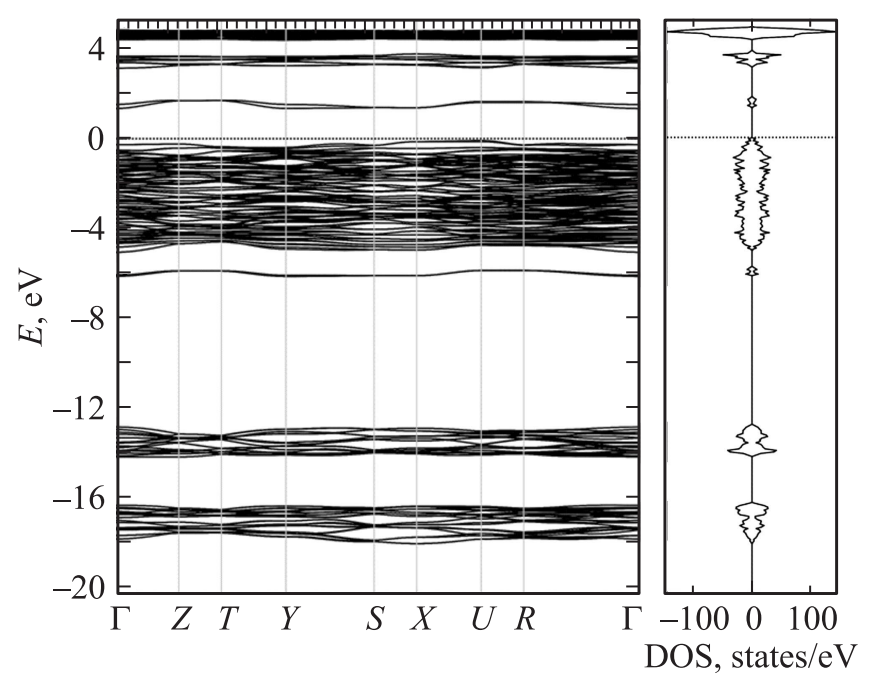

Рис. 4. Электронная зонная структура и плотность состояний кристалла LCTO в магнитоупорядоченном состоянии с удвоенной моноклинной ячейкой.

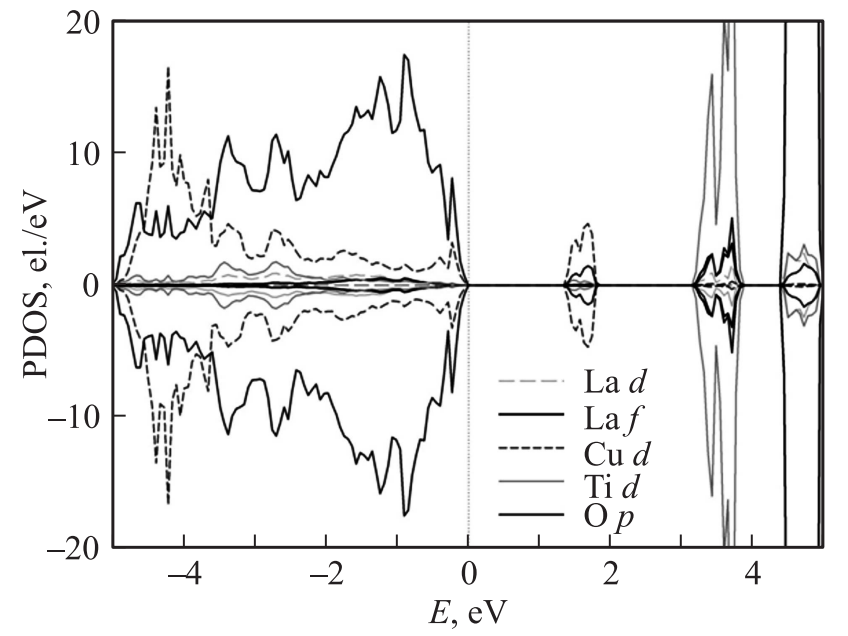

Рис. 5. Разложение парциальных плотностей состояний для $\mathrm{La}, \mathrm{Cu}$, Тi и $\mathrm{O}$ по орбитальным моментам в магнитоупорядоченном состоянии с удвоенной моноклинной ячейкой.

основному магнитному состоянию рассматриваемого соединения с упорядочением магнитных моментов ионов меди два на два, см. далее). На этом же рисунке приведена полная плотность электронных состояний, а разложение парциальных плотностей состояний для $\mathrm{La}$, $\mathrm{Cu}, \mathrm{Ti}$ и $\mathrm{O}$ по орбитальным моментам в моноклинной фазе показано на рис. 5. Из этих рисунков видно, что LCTO в упорядоченной по магнитным моментам меди структуре является диэлектриком с шириной запрещенной зоны около $1.5 \mathrm{eV}$. Валентная зона вблизи уровня Ферми преимущественно занята $d$-электронами меди и титана и $p$-электронами кислорода. Большие пики в зоне проводимости связаны с незанятыми $f$-состояниями лантана и $d$-состояниями титана.

3.3. Магнитная структура и магнитоэлектрически й эффект. Ион двухвалентной меди является магнитным со спином $s=1 / 2$. С целью определения основного магнитного состояния и параметров обменного взаимодействия в LCTO был проведен расчет полных энергий кристалла с учетом спиновой поляризации для различных магнитных упорядоченных структур. Оценка обменных констант взаимодействия была проведена в рамках классической модели Гейзенберга

$$
\mathscr{H}=-\frac{1}{2} \sum_{i, j} J_{i j} \hat{S}_{i} \hat{S}_{j}
$$

где суммирование ведется по всем парам различных узлов магнитной решетки, $J_{i j}-$ константы обменного взаимодействия, $\hat{S}_{i}$ и $\hat{S}_{j}-$ операторы спина на узлах $i$ и $j$ соответственно. Магнитные катионы меди в кубической фазе упорядочены по ГЦК-решетке и, следовательно, каждый атом имеет двенадцать ближайших соседей. В моноклинной фазе эти двенадцать соседей разбиваются на три группы. При этом каждый атом имеет по два соседа вдоль коротких осей $\mathbf{a}$ и $\mathbf{b}$ моно- 
Таблица 3. Выражения для энергий рассматриваемых магнитных структур через обменные константы гамильтониана Гейзенберга и величины энергий этих конфигураций относительно энергии ферромагнитного состояния

\begin{tabular}{c|c|c}
\hline Структура & Выражение для энергии & $\Delta E=E_{i}-E_{\mathrm{F}}, \mathrm{meV}$ \\
\hline $\mathrm{F}$ & $-J_{1}-J_{2}-4 J_{3}$ & 0 \\
$\mathrm{AF} 1$ & $-J_{1}-J_{2}+4 J_{3}$ & 1.4 \\
$\mathrm{AF} 2$ & $-J_{1}+J_{2}$ & -0.4 \\
$\mathrm{AF} 3$ & $J_{1}-J_{2}$ & -2.9
\end{tabular}

клинной ячейки и восемь соседей, расположенных на серединах пространственных диагоналей элементарных ячеек. В связи с этим для определения обменных констант система ионов меди разбивалась на четыре подрешетки. В рамках расчета с учетом спиновой поляризации были вычислены энергии четырех магнитных структур: F - ферромагнитной; AF1 - структуры с ферромагнитным упорядочением в плоскости $(\mathbf{a}, \mathbf{b})$ и антиферромагнитным упорядочением между плоскостями, перпендикулярными длинной оси моноклинной ячейки; AF2 - структуры с ферромагнитным упорядочением магнитных моментов меди в плоскости $(\mathbf{b}, \mathbf{c})$ и антиферромагнитным упорядочением типа два спина вверх, два спина вниз ( $\uparrow \downarrow \downarrow)$ по плоскостям вдоль оси а с удвоением элементарной ячейки вдоль этой оси; AF3 - структуры с ферромагнитным упорядочением магнитных моментов меди в плоскости $(\mathbf{a}, \mathbf{c})$ и анти- ферромагнитным упорядочением типа $\uparrow \uparrow \downarrow \downarrow$ вдоль оси b с удвоением элементарной ячейки вдоль этой оси. Эти магнитные структуры схематически показаны на рис. 6.

В табл. 3 приведены выражения для энергий рассматриваемых магнитных конфигураций через обменные константы гамильтониана (1) и величины энергий этих конфигураций относительно энергии ферромагнитного состояния. Из табл. 3 видно, что наиболее энергетически выгодным в LCTO является состояние AF3. Вычисленные значения обменных констант составили $J_{1}=-1.8 \mathrm{meV}, J_{2}=-0.55 \mathrm{meV}, J_{3}=0.175 \mathrm{meV}$. Оценка парамагнитной температуры Кюри с использованием этих обменных констант дает значение $-27.2 \mathrm{~K}$, что неплохо согласуется с экспериментальным значением $-38 \mathrm{~K}$, полученным для разупорядоченного по катионам титана и меди LCTO [8]. Вычисленный магнитный момент меди равен $0.7 \mu_{\mathrm{B}}$, что также удовлетворительно согласуется с экспериментальным значением $0.9 \mu_{\mathrm{B}}$ [7].

Необходимо отметить, что антиферромагнитные структуры типа $\uparrow \uparrow \downarrow \downarrow$ были обнаружены как экспериментально, так и в результате $a b$ initio расчетов в некоторых соединениях с искаженными структурами перовскита (например, $\mathrm{HoMnO}_{3}$ [13]) и двойного перовскита (например, $\mathrm{Lu}_{2} \mathrm{CoMnO}_{6}$ [14]), содержащих переходные и/или редкоземельные ионы. Особенностью упорядочения типа $\uparrow \uparrow \downarrow \downarrow$ в искаженных структурах перовскита (с пространственной группой $P$ bnm) и двойного перовскита (с пространственной группой $\left.P 2_{1} / n\right)$ является то, что при таком магнитном упорядочении теряется центр инверсии и, следовательно, возникает спонтанная поляризация.

Таблица 4. Изменения относительных координат ионов кристалла, номинальные заряды ионов (e - заряд электрона) и спонтанная поляризация кристалла LCTO с пространственной группой симметрии $P n$ при антиферромагнитном упорядочении типа AF3

\begin{tabular}{c|c|c|c|c|c|c|c|c}
\hline Атом & $\begin{array}{c}\text { Позиция } \\
\text { Уайкова }\end{array}$ & $\begin{array}{c}\text { Заряд, } \\
e\end{array}$ & $\Delta x \cdot 10^{4} / a$ & $\Delta y \cdot 10^{4} / b$ & $\Delta z \cdot 10^{4} / c$ & $\begin{array}{c}P_{x}, \\
\mu \mathrm{C} / \mathrm{m}^{2}\end{array}$ & $\begin{array}{c}P_{y}, \begin{array}{c}P_{z}, \\
\mu \mathrm{C} / \mathrm{m}^{2}\end{array} \\
\mu \mathrm{C} / \mathrm{m}^{2}\end{array}$ \\
\hline La1 & $2 a$ & +3 & -0.1701 & 1.4533 & 0.1773 & & \\
La2 & $2 a$ & +3 & -0.1761 & 1.4535 & 0.1788 & & \\
La3 & $2 a$ & +3 & 0.1861 & 1.0989 & -0.1540 & & \\
La4 & $2 a$ & +3 & 0.1809 & 1.0985 & -0.1539 & & \\
Cu1 & $2 a$ & +2 & -0.0097 & 0.3553 & -0.0702 & & \\
Cu2 & $2 a$ & +2 & -0.0094 & 0.3559 & -0.0701 & & \\
Ti1 & $2 a$ & +4 & -0.0667 & -0.1579 & -0.0951 & & \\
Ti2 & $2 a$ & +4 & -0.0736 & -0.1584 & -0.0963 & & \\
O1 & $2 a$ & -2 & 0.4038 & -0.2185 & -1.2983 & & \\
O2 & $2 a$ & -2 & 0.4076 & -0.2190 & -1.2971 & & \\
O3 & $2 a$ & -2 & -0.3663 & -0.0848 & 1.3025 & & \\
O4 & $2 a$ & -2 & -0.3718 & -0.0840 & 1.3022 & & \\
O5 & $2 a$ & -2 & -1.0273 & 1.2317 & 0.2593 & & \\
O6 & $2 a$ & -2 & -1.0352 & 1.2309 & 0.2588 & & \\
O7 & $2 a$ & -2 & -0.7425 & -2.0209 & -0.1130 & & \\
O8 & $2 a$ & -2 & -0.7506 & -2.0204 & -0.1132 & & \\
O9 & $2 a$ & -2 & 1.1089 & -1.2447 & -0.0566 & & \\
O10 & $2 a$ & -2 & 1.0992 & -1.2457 & -0.0572 & & \\
O11 & $2 a$ & -2 & 0.7108 & 1.8896 & 0.0488 & & \\
O12 & $2 a$ & -2 & 0.7021 & 1.8907 & 0.0476 & & \\
& & & & &
\end{tabular}



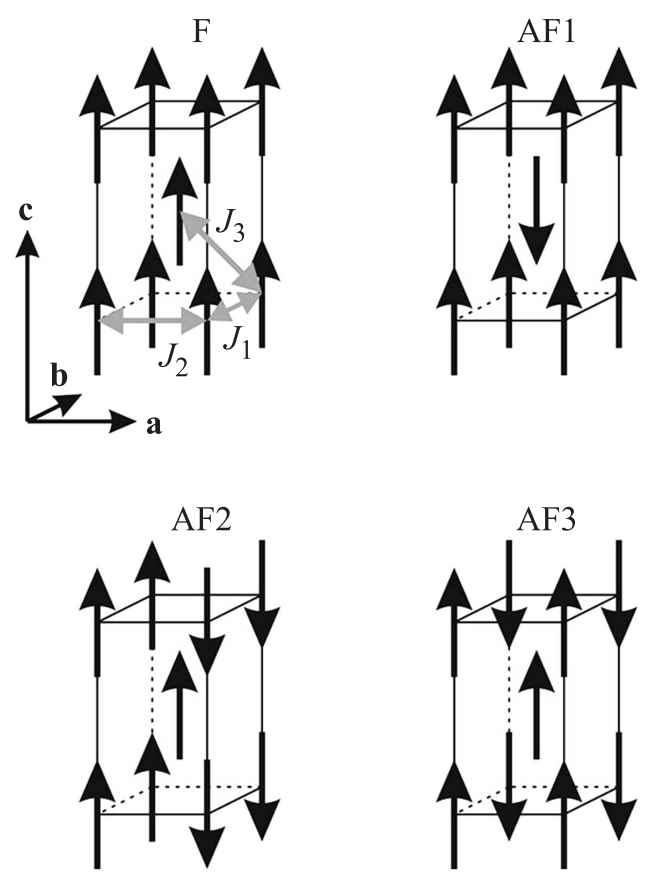

Рис. 6. Схематическое изображение рассматриваемых в настоящей работе магнитных структур кристалла LCTO.

В исследуемом в настоящей работе соединении LCTO упорядочение типа $\uparrow \uparrow \downarrow \downarrow$ магнитных моментов меди вдоль вектора решетки $\mathbf{b}$ моноклинной ячейки приводит к полярной пространственной группе $P n$ и возникновению спонтанной поляризации в плоскости $(\mathbf{b}, \mathbf{c})$. Для оценки величины спонтанной поляризации в кристалле LCTO в фазе $P n$ использовались набор равновесных координат, полученный при релаксации структуры без учета спиновой поляризации, и набор равновесных координат, полученный при релаксации структуры с учетом упорядочения магнитных моментов меди $\uparrow \uparrow \downarrow \downarrow$ (параметры элементарной ячейки в обоих расчетах соответствовали равновесным параметрам удвоенной по параметpy b моноклинной ячейки в расчете с учетом спиновой поляризации). Спонтанная поляризация вычислялась по формуле

$$
P_{i}=\frac{1}{V} \sum_{j=1}^{N} Z_{j} u_{j}^{i},
$$

где $i=x, y, z ; V-$ объем ячейки; $N-$ число атомов в ячейке; $Z_{j}-$ номинальный заряд иона с номером $j$; $u_{j}^{i}-$ смещение иона с номером $j$. Полученные величины смещений ионов и величины компонент поляризации приведены в табл. 4.

\section{4. Заключение}

В рамках теории функционала плотности, реализованной в пакете VASP, было проведено исследование электронных, динамических и магнитоэлектрических свойств двойного перовскита $\mathrm{La}_{2} \mathrm{CuTiO}_{6}$ c „шахматным“ упорядочением катионов меди и титана. Результаты расчета электронной структуры с учетом спиновой поляризации свидетельствуют о том, что данное соединение является диэлектриком. В спектре колебаний решетки высокосимметричной кубической фазы присутствуют нестабильные полярные и антиферродисторсионные моды колебаний. Конденсация наиболее нестабильных антиферродисторсионных мод приводит к возникновению неполярной моноклинной фазы с пространственной группой симметрии $P 2_{1} / n$. Расчет энергий различных магнитных конфигураций в низкосимметричной фазе показал, что в основном магнитном состоянии магнитные моменты меди имеют антиферромагнитное упорядочение типа $\uparrow \uparrow \downarrow \downarrow$ с удвоением параметра $\mathbf{b}$ моноклинной ячейки. При таком упорядочении симметрия кристалла понижается и возникает спонтанная поляризация. Оценка величины спонтанной поляризации дает значение $71 \mu \mathrm{C} / \mathrm{m}^{2}$.

\section{Список литературы}

[1] D. Serrate, J.M.De Teresa, M.R. Ibarra. J. Phys.: Condens. Matter 19, 023201 (2007).

[2] D.G. Franco, R.E. Carbonio, G. Nieva. IEEE Trans. Magn. 49, 4594 (2013).

[3] G. Vaitheeswaran, V. Kanchana, A. Delin. Appl. Phys. Lett. 86, 032513 (2005).

[4] D. Stoeffler, C. Etz. J. Phys.: Condens. Matter 18, 11291 (2006).

[5] S. Gong, P. Chen, B.G. Liu. J. Magn. Magn. Mater. 349, 74 (2014).

[6] M.R. Palacin, J. Bassas, J. Rodriguez-Carvajal, P. GomezRomero. J. Mater. Chem. 3, 1171 (1993).

[7] W.Z. Yang, M.M. Mao, X.Q. Liu, X.M. Chen. J. Appl. Phys. 107, 124102 (2010).

[8] K. Singh, N. Kumar, B. Singh, S.D. Kaushik, N.K. Gaur, S. Bhattacharya, S. Rayaprol, C. Simon. J. Supercond. Nov. Magn. 24, 1829 (2011).

[9] G. Kresse, J. Furthmuller. Phys. Rev. B 54, 11169 (1996).

[10] J.P. Perdew, K. Burke, M. Ernzerhof. Phys. Rev. Lett. 77, 3865 (1996).

[11] S.L. Dudarev, G.A. Botton, S.Y. Savrasov, C.J. Humphreys, A.P. Sutton. Phys. Rev. B 57, 1505 (1998).

[12] A.M. Glazer. Acta Cryst. B 28, 3384 (1972).

[13] A. Munoz, M.T. Casais, J.A. Alonso, M.J. Martinez-Lope, J.L. Martinez, M.T. Fernandez-Diaz. Inorg. Chem. 40, 1020 (2001).

[14] C. Xin, Y. Sui, Y. Wang, Y. Wang, X. Wang, Z. Liu, B. Lib, X. Liu. RSC Adv. 5, 43432 (2015). 